\title{
MODELOS DE FINANCIACIÓN PARA UNA RENTA BÁSICA
}

\section{BASIC INCOME FINANCING MODELS}

\author{
Jordi Arcarons Bullich \\ Universidad de Barcelona, Barcelona, España \\ jordi_arcarons@ub.edu
}

\section{Daniel Raventós Pañella}

Universidad de Barcelona, Barcelona, España

danielraventos@ub.edu

\section{Lluís Torrens Mèlich \\ Ayuntamiento de Barcelona, Barcelona, España \\ LTorrens@bcn.cat}

Palabras clave: Renta básica, Política fiscal, IRPF, Renta Máxima, Impuesto a las grandes fortunas, des-igualdad.

Keywords: Basic income, Fiscal policy, IRPF, Maximum income, Wealth tax, Inequality.

Resumen: En este artículo se ofrece una visión general de cómo podría financiarse una Renta Básica en el Reino de España. Ante todo, se ofrece un repaso de los distintos modelos que se conocen con tal propósito. Se hace hincapié en el tipo de información que permite sustentar dichos modelos, y se pone especial énfasis en las ventajas e inconvenientes que puede generar la utilización de dicha información. Se describen los principales análisis que se desprenden de la aplicación de dichos modelos de financiación. Se justifica una reforma tributaria del IRPF, eje central de la financiación de la propuesta de Renta Básica, junto con una propuesta de implementación de un impuesto sobre la riqueza, que complementaría esa financiación. Por último, a través de un supuesto concreto se analiza los efectos sobre: la desigualdad y la redistribución de la renta, la pobreza y los grupos de ganadores y perdedores, al comparar la situación sin modelo de Renta Básica (ex-ante) y con modelo de Renta Básica (ex-post).

Abstract: This article provides an overview of how a Basic Income could be financed in the Kingdom of Spain. First of all, it offers a review of the different models known for this purpose. Emphasis is placed on the type of information which allows these models to be supported, and special focus is placed on the advantages and disadvantages which can be generated by the use of this information. The main analyses arising from the application of these financing models are described. A reform of personal income tax, the central axis of the 
financing of the Basic Income proposal, is justified, together with a proposal for the implementation of a wealth tax, which would complement this financing. Finally, through a concrete assumption, the effects on inequality and redistribution of income, poverty and groups of winners and losers are analysed by comparing the situation without the Basic Income model (exante) and with the Basic Income model (ex-post).

\section{Introducción}

De la misma manera que se repiten determinados mantras del tipo "un estado es como una familia y no puede mantener una deuda durante mucho tiempo" o "lo importante es contener la inflación", hay uno que es constante: la Renta Básica no se puede financiar o es muy cara. Nosotros pensamos que no es así. Pero pensar sin aportar razones y evidencias es un brindis al sol. En este artículo aportamos razones para mostrar cómo se puede financiar una Renta Básica. Pero quizás sea necesario dar un paso atrás y explicar parte de lo que fundamenta nuestra exposición. Una Renta Básica formaría parte de una política económica. Una política económica se compone de muchas medidas de tipo fiscal, monetario, laboral... La Renta Básica pretende dar respuesta al hecho de que existen centenares de millones de personas en todo el mundo y una decena larga en el Reino de España que no tienen la existencia material garantizada, que no tienen las necesidades mínimas cubiertas, que no tienen una vida que remotamente se pueda llamar digna porque tienen que mendigar subsidios 0 trabajos mal pagados. Personas que no pueden aguantar el "test de la mirada" como lo denomina Pettit (2012) porque tienen que bajar sumisamente los ojos ante las miradas de los que deciden sus vidas porque de ellos depende su puesto de trabajo o su subsidio. Es gente que no es libre ni puede serlo porque depende de la arbitrariedad de otros. Ante estas situaciones sí que la Renta Básica puede aportar mucho. Puede ayudar que las vidas de estas personas sean más libres o no tan subordinadas como lo son ahora. Pero la Renta Básica no puede aportar soluciones a las grandes concentraciones de poder privado, ni a las legislaciones permisivas con la elusión y el fraude fiscales, ni a la gran desigualdad en muchas realidades entre hombres y mujeres, ni al problema de la vivienda. Son realidades que precisan de políticas específicas. Pero sí que la Renta Básica es una medida contra la pobreza y por la garantía de la existencia material a toda la población. Es una medida pues necesaria para la libertad de todos y todas puesto que no puede haber libertad sin las condiciones materiales de existencia cubiertas.

Nuestro objetivo en este artículo es mostrar algo muy concreto en contra del mantra al que nos referíamos al principio según el cual no se puede financiar la Renta Básica. Se puede y lo que mostramos es una forma de hacerlo.

Varios trabajos de algunos miembros de la Red Renta Básica (RRB) desde hace ya unos cuantos años se elaboran en forma de modelos que muestran como financiar una Renta Básica (RB). Históricamente, estos modelos ${ }^{1}$ se han basado en infor-

1. La descripción de dichos modelos puede consultarse en Arcarons, Raventós y Torrens (2017b), Gimeno (2015) y Díaz, Gimeno y Gómez (2019). En el ámbito internacional puede 
mación proveniente de la Agencia Estatal de la Administración Tributaria (AEAT) organismo que elabora una muestra anual de declarantes del IRPF desde el año $2003^{2}$. Más recientemente, también se han propuesto otros modelos ${ }^{3}$ para financiar una RB, que utilizan la información que proporciona la Encuesta de Condiciones de Vida (ECV) 4 que elabora el Instituto Nacional de Estadística (INE).

Respecto a las dos fuentes de información antes señaladas y que utilizan los distintos modelos propuestos, es conveniente efectuar algunas precisiones en lo concerniente a las conclusiones y a las limitaciones que pueden extraerse de las mismas.

La información que proporciona la AEAT tiene varios elementos favorables. En primer lugar, un elevado tamaño de la muestra, que en los sucesivos años disponibles ha ido variando, pero que siempre ha superado los 2 millones de registros, garantizando una muy amplia representatividad de la cola superior de la distribución de renta, aspecto al que más adelante nos

consultarse Ortiz et alters (2018) y Gentilini et alters (2020).

2. Aunque los microdatos fiscales referidos no pueden obtenerse directamente de la AEAT, una descripción del tipo de información y del período histórico al que se tiene acceso, puede consultarse en: https:/www.agenciatributaria.es/AEAT. internet/datosabiertos/catalogo/hacienda/Estadistica_de_los_declarantes_del_IRPF.shtml

3. La descripción de este otro tipo de modelos puede consultarse en Arcarons, Raventós y Torrens (2020). También Granell y Fuenmayor (2019) elaboran un modelo de Renta Básica utilizando este tipo de información.

4. El INE permite descargar los microdatos de la ECV del año 2008 al año 2019 desde: https:// www.ine.es/dyngs/INEbase/es/operacion.htm?c $=$ Estadistica_C\&cid=1254736176807\&menu=re sultados\&secc $=1254736195153 \&$ idp $=12547359$ 76608\#!tabs-1254736195153 referiremos. En segundo lugar, un desglose de la renta de la persona contribuyente según sus rendimientos del trabajo, del capital mobiliario, inmobiliario, atribuciones de rentas y ganancias y pérdidas patrimoniales; ofreciendo, por tanto, todas las fuentes de ingresos que constituyen la renta bruta que percibe la persona. Por último, la información sociofamiliar (edad, género, estado civil) de la persona declarante y también de las personas dependientes de su declaración, de las que puede obtenerse, además, la relación de parentesco. En el lado negativo, también hay que apuntar algunos inconvenientes. El primero, la unidad representativa es la persona declarante y no puede identificarse a partir de ella el hogar o unidad de convivencia, lo que afecta a los hogares con varios declarantes que hacen declaraciones individuales. El segundo, la información se refiere a lo que se denomina territorio fiscal común (TFC) que excluye los datos de las comunidades forales del País Vasco y de Navarra. En tercer lugar, puesto que se trata de datos administrativos, no ofrece ninguna otra información (más allá de lo apuntado más arriba como características sociofamiliares), relativa a las condiciones sociales de la persona contribuyente ni de las personas dependientes de la misma. Y, en cuarto lugar, que la muestra no recoge datos de contribuyentes no obligados a presentar declaración por debajo de 10.000 euros anuales de ingresos (los no obligados que están entre 10.000 y 22.000 sí que son recogidos en una submuestra específica).

Si nos centramos en la información que contiene la ECV hay que destacar también elementos favorables. En primer lugar, la referencia es el hogar o unidad de convivencia y, además, se facilita información sobre las rentas que perciben todos sus 
integrantes, y que ha sido contrastada por el INE con datos de la AEAT. En segundo lugar, es una información representativa de todo el Reino de España y puede considerarse que también lo es, aunque con ciertos límites, a nivel de comunidad autónoma. Por último, puesto que se trata de una encuesta, aporta información muy detallada relativa a las condiciones sociales de todas las personas integrantes del hogar. En el lado negativo, destacan fundamentalmente dos cuestiones. El tamaño muestral no es elevado, pese a representar a más de 18 millones de hogares, lo que conlleva que el extremo superior de la distribución de la renta no esté todo lo bien representado que debería esperarse ${ }^{5}$. Y, también es una limitación el hecho de que no vienen recogidas una parte muy significativa de las rentas especulativas -como las plusvalías-, las que forman parte de la base del ahorro en el IRPF.

De las anteriores enumeraciones de ventajas e inconvenientes, se desprende que cuando se utiliza una $u$ otra fuente de información, deberán asumirse ciertas restricciones. La primera, con los datos administrativos que proporciona la AEAT,

5. Tomando como referencia los años 2017 y 2018, los hogares encuestados fueron 13.740 (34.911 personas) y 13.368 (33.734 personas), que deben representar, respectivamente, más de 18 millones de hogares y más de 46 millones de personas. Otro dato significativo, en 2018 la $\mathrm{ECV}$, que proporciona rentas correspondientes a 2017, tiene una clara limitación en la cola superior de su distribución de renta, puesto que no recoge los grandes contribuyentes del Reino de España, ya que no hay ni un solo hogar con más de 480.000 euros de renta, cuando según la AEAT en 2016 (siempre en lo relativo al TFC, excluyendo el País Vasco y Navarra) ya hubo más 12.000 contribuyentes por encima de esa cifra. el cómputo del ahorro en prestaciones, que tiene como consecuencia la implantación de la RB, debe calcularse de forma agregada y no puede evaluarse individualmente como sí permiten los datos procedentes de la ECV. La segunda, es que usando la ECV, deberán imputarse las rentas especulativas puesto que no aparecen descritas ${ }^{6}$, cuestión que no se plantea con la información que suministra la AEAT. La tercera, los datos administrativos son más robustos desde el punto de vista de la distribución de renta de los más ricos, mientras que los que facilita la ECV están limitados en el caso de las rentas de los más ricos. Y a la inversa en el caso de los más pobres, aunque la AEAT dispone de estadísticas adicionales como la del Mercado de Trabajo y Pensiones en las Fuentes Tributarias para estimar los ingresos agregados de la cola inferior. La cuarta, los análisis posteriores del impacto redistributivo, mejora de la desigualdad y erradicación de la pobreza pueden efectuarse con mejor precisión a partir de la información de la ECV, puesto que la unidad de referencia es el hogar. Por último, los datos de la AEAT no incluyen las comunidades forales, que por el contrario sí lo están en la ECV.

Las características comunes de todos los modelos, basados en cualquiera de los dos tipos de información a los que han sido referidos, pueden resumirse en lo siguiente:

1) Propuesta de una cantidad para la RB que permita garantizar las condicio-

6. En el caso del año 2018, la cifra imputada agregada es muy cercana a los 24.000 millones de euros, lo que representa incrementar un $4,17 \%$ la renta bruta que aparece en la ECV. 
nes materiales para la existencia de las personas ${ }^{7}$.

2) La RB sustituye a toda otra cantidad económica que se perciba por parte del Estado, siempre que dicha cantidad sea inferior a la RB; pero si es superior, la parte que la supera se seguirá percibiendo en las mismas condiciones.

3) La RB no estará gravada por el IRPF.

4) La financiación de la RB se consigue fundamentalmente mediante una reforma del IRPF en la que pueden colaborar también la introducción y/o reformas de otras figuras impositivas.

5) Para financiar la RB, no se debe detraer ninguna cantidad de lo que recauda el estado vía IRPF, para mantener los pilares fundamentales de su inversión social como sanidad y educación.

El tipo de análisis que permite cualquiera de estos modelos puede resumirse en:

1) Determinar el nivel de desigualdad existente entre la situación inicial y final, esto es: cuál es la distribución de renta sin RB (ex-ante) y con RB (expost).

2) Evaluar el grado de progresividad que se consigue mediante la reforma del IRPF propuesta para financiar la RB, frente al del actual IRPF.

3) Calcular el nivel de redistribución conseguido al aplicar la RB.

4) Analizar por grupos de renta, cuáles resultan ganadores y perdedores.

7. Siguiendo la definición que se propone desde la RRB, generalmente esta cantidad se asimila al umbral de pobreza, que se define como el $60 \%$ de la mediana de la renta neta equivalente de los hogares.
5) Calcular el coste que deben asumir los más ricos, para sufragar la financiación de la RB.

\section{Resultados}

En lo concerniente a los modelos que utilizan la información fiscal y administrativa de la AEAT se remite al lector o lectora a los trabajos de Arcarons, Raventós y Torrens (2013a, 2013b, 2016), específicos de una financiación para el caso de Catalunya y para el ámbito del Reino de España a Arcarons, Domènech, Raventós y Torrens (2014). En cualquier caso, hay que destacar que sus principales conclusiones son:

1) con una reforma fiscal basada en el IRPF, es posible financiar una RB para toda la población por una cantidad muy parecida al umbral de pobreza de los períodos de referencia

2) se consigue que el $70 \%$ de la población ordenada según renta resulte beneficiada (ganadora) con dicha reforma

3) hay una mejora notable en la desigualdad (muy importante disminución del índice de Gini), acompañada de un significativo incremento de la progresividad del IRPF y de su efecto redistributivo

4) el coste de transferencia de ricos (sobre los que recae el peso de la financiación) a pobres se cifra en unos 35.000 millones de euros $(3,5 \% \text { del PIB })^{8}$.

8. Este último dato, que se obtiene en el posterior de los trabajos citados para el global del Reino de España, desmiente con rotundidad las torpes interpretaciones relativas a que el coste de la RB es sencillamente multiplicar la cantidad transferida por todas las personas que vayan a recibirla, siendo muy ilustrativo al respecto el artículo de 


\section{Cuadro 1}

\begin{tabular}{|c|c|c|}
\hline $\begin{array}{r}\text { Hogares en la muestra } \\
\text { Hogares en la población } \\
\text { Personas en la muestra } \\
\text { Personas en la población } \\
\text { De } 0 \text { a } 13 \text { años } \\
\text { De } 14 \text { a } 17 \text { años } \\
\text { De } 18 \text { y más años }\end{array}$ & $\begin{array}{r}13.368 \\
18,55 \\
33.734 \\
46,18\end{array}$ & $\begin{array}{r}6,07 \\
1,78 \\
38,33\end{array}$ \\
\hline $\begin{array}{r}\text { Renta Bruta } \\
\text { Cotizaciones a la SS } \\
\text { IRPF ex-ante } \\
\text { Impuesto sobre el patrimonio } \\
\text { Transferencias a otros hogares } \\
\text { Renta Neta ex-ante }\end{array}$ & $\begin{array}{r}660.459,34 \\
548.329,87\end{array}$ & $\begin{array}{r}27.812,39 \\
79.957,05 \\
432,31 \\
3.927,72\end{array}$ \\
\hline $\begin{array}{r}\text { Indice de Gini sobre Renta Bruta } \\
\text { Indice de Gini sobre Renta Neta ex-ante } \\
\text { Indice de Suits sobre IRPF ex-ante } \\
\text { Efecto redistributivo ex-ante }\end{array}$ & $\begin{array}{l}0,3836 \\
0,3457 \\
0,3369 \\
0,0380\end{array}$ & \\
\hline $\begin{array}{r}\text { Indice de pobeza de Sen } \\
\% \text { Hogares en riesgo de pobreza } \\
\% \text { Personas(<18 años) en riesgo de pobreza } \\
\% \text { Personas(>=18 años) en riesgo de pobreza } \\
\% \text { Hogares en riesgo de pobreza severa } \\
\text { \% Personas(<18 años) en riesgo de pobreza severa } \\
\% \text { Personas(>=18 años) en riesgo de pobreza severa }\end{array}$ & $\begin{array}{c}0,0971 \\
19,28 \% \\
25,71 \% \\
19,51 \% \\
8,46 \% \\
11,56 \% \\
8,09 \%\end{array}$ & \\
\hline
\end{tabular}

Fuente: ECV-2018 y elaboración propia. Valores de población en millones de personas. Valores monetarios en millones de euros.

Se expone con detalle uno de los dos supuestos más relevantes del último de los modelos publicados en el trabajo ya citado de Arcarons, Raventós y Torrens (2020), que utiliza la publicación por parte del INE de los microdatos correspondientes a la ECV de 2018 .

El cuadro 1 proporciona algunos de los resultados más relevantes que se derivan de la ECV-2018, y que serán el punto de comparación con el supuesto de RB que se va a analizar. Conviene destacar que las dos cifras de Renta bruta y neta ex-ante (denominadas así para indicar la situación previa

Arcarons, Raventós y Torrens (2017a), en donde se pone énfasis en el carácter redistributivo de cualquier modelo de financiación de la RB basado en un reforma fiscal del IRPF.

9. En el momento de redactar este artículo se han puesto a disposición del público los microdatos relativos a 2019, que obviamente no han podido ser utilizados aquí. a la implantación de la RB) ya están incrementadas con la imputación de las rentas procedentes de variaciones patrimoniales que no están reflejadas en la ECV ${ }^{10}$.

Respecto a la desigualdad de la distribución de renta, la hemos medido a través del índice de Gini ${ }^{11}$, el grado de progresividad del IRPF (índice de Suits) y el efecto redistributivo alcanzado mediante el IRPF. Para estos dos últimos casos, es notorio que pese a observarse un grado de progre-

10. Ver la anterior nota a pie de página 6 .

11. Según los datos de Eurostat, a través de la operación UE-SILC que establece una metodología común para la ECV efectuada en 36 países, el índice de Gini sobre la renta neta equivalente para el Reino de España del año 2017 (los valores de rentas de la ECV siempre se refieren al año anterior), lo sitúa en la posición 29 ordenados de menor a mayor desigualdad; tan solo detentan mayor desigualdad Letonia, Montenegro, Lituania, Serbia, Bulgaria, Turquía y Albania. 
sividad ciertamente elevado (0,3369), el efecto redistributivo es muy poco notable, puesto que después de aplicar el IRPF, la desigualdad solo se consigue disminuir en un escaso 3,8\%. Finalmente, se muestran los indicadores ${ }^{12}$, ciertamente preocupantes, relativos a la situación de pobreza.

En relación al supuesto de financiación de RB, la primera cuestión es la determinación de la cantidad individual de RB que va a percibir cada persona. Se utiliza el umbral de pobreza que determina la ECV, esto es 8.815 euros anuales para una persona adulta, pero la cantidad percibida por el hogar se calcula según la escala de equivalencia modificada de la OCDE ${ }^{13}$. Esta escala asigna el valor 1 para la primera persona del hogar de 18

12. El índice de pobreza de Sen es bastante similar al índice de Gini, pero calculado sobre los hogares declarados en riesgo de pobreza. Se considera un hogar en riesgo de pobreza aquél cuya renta neta equivalente está por debajo del umbral de pobre$\mathrm{za}$; mientras que una persona pobre es cualquier integrante de ese hogar. El calificativo de pobreza severa conlleva que el cálculo del umbral de pobreza sea más extremo, rebajándolo al $40 \%$ de la mediana de renta equivalente que se obtiene de la distribución. Por último, el concepto de carencia material severa es un concepto subjetivo, que obedece a privaciones materiales del hogar. Se consideran hasta 9 distintas privaciones, entre las que destacan: afrontar gastos imprevistos, ir de vacaciones al menos una semana al año, permitirse una comida cada dos días con carne, pollo o pescado o equivalente para personas vegetarianas, mantener la vivienda a temperatura adecuada. Cuando 4 de esas 9 privaciones existen, el hogar se considera en carencia material severa.

13. Esta escala simplemente quiere constatar la evidencia según la cual vivir en un mismo hogar más de una persona es más caro que lo que costaría vivir una sola persona, pero en una proporción decreciente. Cuatro personas viviendo en el mismo hogar no es cuatro veces más caro que lo que representa vivir una por distintas razones, una de ellas es por los costes fijos que supone vivir en un mismo hogar y por los que no son exactamente o más años, 0,5 para el resto con 14 o más años y 0,3 para las menores de 14 años. La RB de un hogar se distribuiría y pagaría equitativamente de forma individual entre las personas adultas, y entre los tutores legales, la parte de los menores de edad. Esta es una consideración importante: no hay un cabeza de hogar (sea mujer u hombre) que reciba toda la renta. Así, un hogar con 3 personas mayores de 18 años, una de ellas descendiente de las otras 2, y otra menor de 14, supondría una escala de equivalencia de 2,3 . Esto significa que este hogar recibiría una RB multiplicada por 2,3 (20.275 euros) que a su vez se dividiría equitativamente entre estas 4 personas (5.069 euros cada uno), aunque a efectos prácticos se dividiría entre las dos personas adultas (7.603 euros cada uno) si tuvieran la potestad de los menores de 18 años como acostumbra a suceder y para la tercera persona adulta descendiente (5.069 euros). En el cuadro 2 puede observarse qué cantidad percibe el hogar según su composición de personas, calculado con el criterio de hogar-OCDE.

En cualquier caso, hay que hacer hincapié en que siempre se trata de una transferencia individual a las personas, aunque la determinación de la cantidad se hace previamente considerando las características del hogar o unidad de convivencia de la persona, siendo finalmente la RB percibida individualmente por cada uno de los integrantes del hogar.

Una vez determinada y justificada la cantidad de RB, el paso siguiente es explicar cómo va a financiarse ${ }^{14}$. Previamente hay

proporcionales a la cantidad de personas: alquiler, electricidad, agua...

14. En el Glosario, se expresan algunas definiciones para comprender el funcionamiento del modelo de financiación. 


\section{Cuadro 2}

\begin{tabular}{|c|c|c|c|c|c|c|c|c|c|c|c|c|c|c|}
\hline & $1 \mathrm{~A}$ & $1 \mathrm{~A}+1 \mathrm{M}$ & $1 A+2 M$ & $1 A+3 M$ & $2 A$ & $2 A+1 M$ & $2 A+2 M$ & $2 A+3 M$ & $2 A+4 M$ & $3 A$ & $3 A+1 M$ & $3 A+2 M$ & $3 A+3 M$ & $3 A+4 M$ \\
\hline eq & 1 & 1,3 & 1,6 & 1,9 & 1,5 & 1,8 & 2,1 & 2,4 & 2,7 & 2 & 2,3 & 2,6 & 2,9 & 3,2 \\
\hline (1) & 8.815 & 11.460 & 14.104 & 16.749 & 13.223 & 15.867 & 18.512 & 21.156 & 23.801 & 17.630 & 20.275 & 22.919 & 25.564 & 28.208 \\
\hline (2) & 8.815 & 5.730 & 4.701 & 3.526 & 6.611 & 5.289 & 4.628 & 4.231 & 3.967 & 5.877 & 5.069 & 4.584 & 4.261 & 4.030 \\
\hline \multirow{3}{*}{ (3) } & 8.815 & 11.460 & 14.104 & 16.749 & 6.611 & 7.934 & 9.256 & 10.578 & 11.900 & 5.877 & 7.603 & 9.168 & 10.651 & 12.089 \\
\hline & --- & --- & --- & --- & 6.611 & 7.934 & 9.256 & 10.578 & 11.900 & 5.877 & 7.603 & 9.168 & 10.651 & 12.089 \\
\hline & --- & --- & --- & --- & --- & --- & --- & --- & --- & 5.877 & 5.069 & 4.584 & 4.261 & 4.030 \\
\hline
\end{tabular}

Cantidades percibidas por el hogar (1), por cada una de las personas del hogar (2) y por las personas mayores de 18 años del hogar (3), según la composición de adultos (A) y menores (M), a partir del umbral de pobreza de la ECV-2018 de 8.815 euros.

Se supone cuando hay adultos y menores que el primer y/o segundo adulto son los tutores de los menores y que en el caso de más de 2 adultos, el tercero es descendiente mayor de 18 años.

que indicar tres cuestiones importantes sobre el modelo de financiación.

La primera de ellas es el ahorro por implantación de la RB, relativa a que la RB transferida elimina cualquier prestación monetaria recibida por el estado, cuando ésta es inferior; pero en el caso de que esa prestación sea más elevada, la cantidad que supere la RB seguirá percibiéndose en las mismas condiciones. Así, todas las pensiones no contributivas, que generalmente se situarán por debajo de la RB, y una parte de las pensiones contributivas, a las que deberá añadirse también las prestaciones por desempleo, desaparecen y conforman un ahorro, que deberá tenerse en cuenta para la determinación del coste de financiación.

La segunda cuestión, se refiere a la reforma del IRPF con cuya recaudación se debe garantizar la financiación de la RB y además lo que ya se obtenía con el IRPF previo, como condición de que con la RB no se va a detraer ni un solo euro de lo que el estado destina a inversión social como sanidad y educación. Aquí debe decirse que se trata de una absoluta simplificación respecto al complejo esquema de liquidación actualmente existente: se eliminan las reducciones y compensaciones previas a la determinación de la base imponible, se eliminan los mínimos personales y familiares, se elimina el concepto de base imponible del ahorro y todos los rendimientos tributan a una única tarifa o tipo único ${ }^{15}$ y se eliminan también todas las deducciones sobre la cuota.

Por último, la tercera cuestión es que se contempla una cláusula de compensación o status quo para aquellos hogares que se sitúan por debajo del 80 percentil de la renta bruta equivalente ${ }^{16}$. Esto significa que cualquier hogar por debajo del $20 \%$ más rico (el de mayor renta), nunca empeora su nivel de ingresos netos respecto a la situación previa a la reforma (en adelante ex-ante) ${ }^{17}$.

15. En Arcarons, Raventós y Torrens (2017b) se discute ampliamente sobre la posibilidad de trabajar con un tipo único o con una tarifa por tramos. La adopción de un tipo único en el IRPF, siempre combinado con la transferencia de RB se demuestra como una opción muy progresiva desde el punto de vista fiscal, pero, además, muy pedagógica para entender y ejemplificar el esquema de RB que se propone; por esta razón, los resultados del supuesto analizado se basan en esta opción del tipo único. En el mismo trabajo arriba señalado se pueden consultar resultados que utilizan la tarifa por tramos.

16. El resultado de dividir la Renta Bruta del hogar entre su escala de la OCDE modificada.

17. Dos ejemplos significativos entre los que se citan en Arcarons, Raventós y Torrens (2020): para 


\section{Cuadro 3}

\begin{tabular}{|c|c|}
\hline Concepto & \\
\hline Renta Básica según supuesto & $273.760,05$ \\
\hline Ahorro por implantación RB & $82.890,26$ \\
\hline IRPF ex-post & $270.813,28$ \\
\hline Tipo único de financiación & $46,83 \%$ \\
\hline Renta Neta ex-post (antes compensación) & $548.343,42$ \\
\hline Renta Neta ex-post & $563.434,72$ \\
\hline Redistribución entre hogares (mediante IRPF ex-post y Renta Bàsica) & $35.548,95$ \\
\hline Coste compensación (hogares compensados incluyendo el percentil 80) & $15.106,85$ \\
\hline Redistribución (mediante IRPF ex-post, Renta Bàsica y compensación) & $50.655,80$ \\
\hline$\%$ s /PIB & $4,33 \%$ \\
\hline Índice de Gini sobre Renta Neta ex-post & 0,2359 \\
\hline Índice de Suits sobre IRPF ex-post & 0,2293 \\
\hline Índice de Suits sobre Renta Básica & $-0,2359$ \\
\hline Efecto redistributivo ex-post & 0,1478 \\
\hline \% Hogares ganadores & $51,05 \%$ \\
\hline \% Hogares compensados & $29,74 \%$ \\
\hline$\%$ Hogares no perdedores & $80,79 \%$ \\
\hline \% Hogares perdedores & $19,21 \%$ \\
\hline Índice de Sen ex-post & 0,0023 \\
\hline$\%$ Hogares en riesgo de pobreza & $0,53 \%$ \\
\hline$\%$ Hogares en riesgo de pobreza severa & $0,15 \%$ \\
\hline$\%$ Personas(<18 años) en riesgo de pobreza & $0,44 \%$ \\
\hline$\%$ Personas(<18 años) en riesgo de pobreza severa & $0,18 \%$ \\
\hline$\%$ Personas(>=18 años) en riesgo de pobreza & $0,45 \%$ \\
\hline$\%$ Personas(>=18 años) en riesgo de pobreza severa & $0,13 \%$ \\
\hline
\end{tabular}

Fuente: ECV-2018 y elaboración propia

Valores monetarios en millones de euros

En el cuadro 3 se resumen los principales resultados obtenidos para el supuesto analizado, en donde destacan:

- $19,2 \%$ de hogares perdedores y un $80,8 \%$ de no perdedores.

- Tipo único del 46,83\% para el nuevo IRPF sobre todas las rentas que garantizaría la financiación.

- Índice de Gini de 0,2359 frente al 0,3836 sobre la Renta bruta, reduciéndose cerca de 15 puntos y alcanzando uno de los niveles más igualitarios del mundo.

- Porcentaje de hogares en riesgo de pobreza y pobreza severa, que práctica-

hogares unifamiliares por debajo de 29.592 euros anuales o, para hogares compuestos por 2 personas mayores de 18 años y 2 menores, a los que correspondería una renta bruta equivalente de 62.142 euros, o una renta bruta familiar (escala de equivalencia de 2,1) algo por encima de los 130.000 euros, ninguno resultaría perjudicado con la reforma. mente desaparecen, 0,54\% y 0,15\%, frente al $19,28 \%$ y $8,46 \%$ que se obtienen en la ECV.

- Efecto redistributivo del 14,78\%, frente al 3,8\% que se obtiene en la situación ex-ante.

- El coste para los hogares perdedores (los que su renta disponible neta después de cobrar la RB y pagar el nuevo IRPF es inferior a la renta real que tuvieron disponible en el 2017) es de 35.549 millones de euros.

- La compensación para que ningún hogar hasta el percentil 80 de Renta Bruta equivalente resulte perjudicado, supone un coste adicional de 15.107 millones de euros ${ }^{18}$; teniendo en cuenta que la gran mayoría, el $80 \%$, de los hogares compensados son de pensionistas (lo

18. En el siguiente epígrafe se hace una propuesta de cómo podría financiarse este coste adicional. 


\section{Cuadro 4. Tipos efectivos}

\begin{tabular}{crr} 
Percentiles & $\begin{array}{c}\text { Tipo efectivo } \\
\text { ex-ante }\end{array}$ & $\begin{array}{c}\text { Tipo efectivo } \\
\text { ex-post }\end{array}$ \\
\hline $\mathbf{1 0 \%}$ & $2,18 \%$ & $-282,46 \%$ \\
$\mathbf{2 0 \%}$ & $1,18 \%$ & $-139,58 \%$ \\
$\mathbf{3 0 \%}$ & $1,86 \%$ & $-85,44 \%$ \\
$\mathbf{4 0 \%}$ & $3,42 \%$ & $-46,88 \%$ \\
$\mathbf{5 0 \%}$ & $5,29 \%$ & $-24,14 \%$ \\
$\mathbf{6 0 \%}$ & $7,05 \%$ & $-8,90 \%$ \\
$\mathbf{7 0 \%}$ & $9,96 \%$ & $1,59 \%$ \\
$\mathbf{7 5 \%}$ & $11,37 \%$ & $8,29 \%$ \\
$\mathbf{8 0 \%}$ & $12,18 \%$ & $12,70 \%$ \\
$\mathbf{8 5 \%}$ & $14,20 \%$ & $16,76 \%$ \\
$\mathbf{9 0 \%}$ & $15,15 \%$ & $20,55 \%$ \\
$\mathbf{9 5 \%}$ & $16,92 \%$ & $25,19 \%$ \\
$\mathbf{9 8 \%}$ & $19,64 \%$ & $30,24 \%$ \\
$\mathbf{1 0 0 \%}$ & $24,85 \%$ & $38,91 \%$
\end{tabular}

Fuente: ECV-2018 y elaboración propia

cual reduce los riesgos de crear trampas de pobreza, como las que generan las rentas mínimas como el IMV o las autonómicas).

- El coste total para los ricos sería pues de 50.656 millones de euros, el 4,33\% del PIB.

Por último, el cuadro 4 y el gráfico 1 permiten representar los tipos efectivos (lo realmente tributado) ex-ante y ex-post para ilustrar el grado de progresividad que se obtiene en ambas situaciones.

\section{Impuesto sobre la riqueza}

Tal como hemos señalado anteriormente, la financiación de la Renta Básica, en todos los modelos que se han mencionado se consigue fundamentalmente a través de una reforma del actual IRPF; no obstante, existen claros argumentos para introducir fuentes complementarias de financiación adicional. De entre todas las posibles, destaca la imposición sobre la

\section{Gráfico 1}

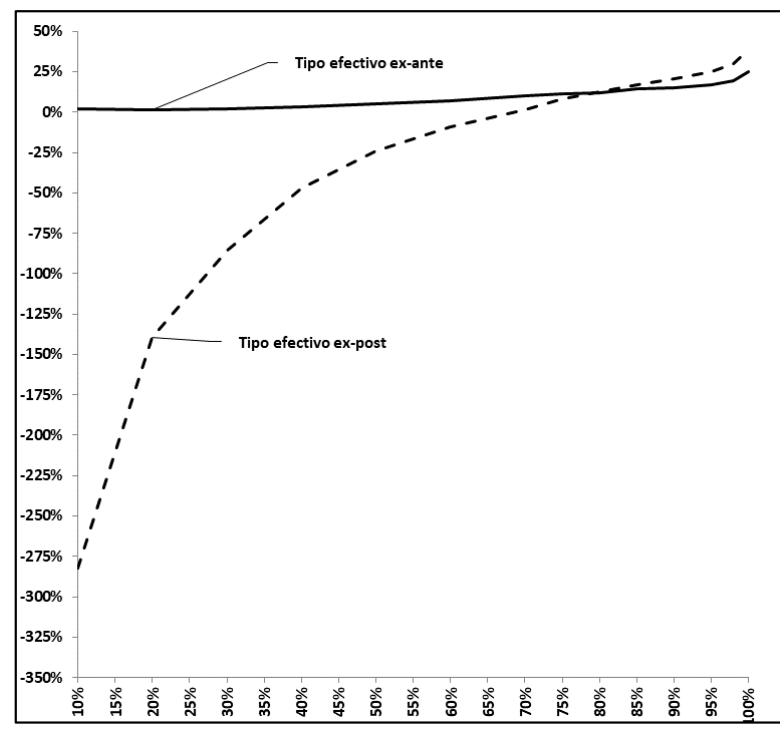

riqueza ${ }^{19}$. A este respecto, se puede aportar la siguiente información, que recogen los cuadros 5 y 6 , a partir de la Encuesta Financiera de las Familias que elabora cada tres años el Banco de España ${ }^{20}$.

Según se indica en los cuadros 5 y 6 , el cruce del percentil 90-100 de mayor riqueza y del percentil 90-100 de mayor renta, acumula las nada despreciables cifras de más de 0,96 billones de euros de riqueza (descontando la vivienda habitual) y de prácticamente 0,085 billones

19. Ver por ejemplo: Piketty (2019), Bertomeu y Raventós (2020), Franco et. alters (2015), Kucharz (2020) o el manifiesto "Millionaires for Humanity" en https://www.millionairesforhumanity.com/. Un impuesto complementario a las grandes fortunas, tendría un efecto moderador en la reforma propuesta del IRPF, rebajando la presión fiscal establecida sobre este último.

20. https://www.bde.es/webbde/es/estadis/infoest/ temas/te_encuestas.html. La última publicación con microdatos es de 2014. Actualmente el Banco de España ha publicado ya su informe correspondiente a la versión de 2017, pero no los microdatos correspondientes para este año. 


\section{Cuadro 5. Distribución de la renta y la riqueza en el Reino de España. Valores de riqueza en millones de euros.}

\begin{tabular}{|c|c|c|c|c|c|c|c|c|c|c|c|c|c|c|}
\hline \multicolumn{15}{|c|}{ PERCENTILES DE RIQUEZA } \\
\hline \multirow{14}{*}{ 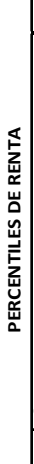 } & & $<10 \%$ & $10-20 \%$ & $20-30 \%$ & $30-40 \%$ & $40-50 \%$ & $50-60 \%$ & $60-70 \%$ & $70-80 \%$ & $80-90 \%$ & $90-95 \%$ & $95-98 \%$ & $98-100 \%$ & Total \\
\hline & $<10 \%$ & $-267,4$ & $-2.235,5$ & $3.565,4$ & $3.255,8$ & $1.436,6$ & $1.774,3$ & $4.743,3$ & $2.995,9$ & $4.364,1$ & $4.992,0$ & $4.489,4$ & $6.262,1$ & $35.375,8$ \\
\hline & $10-20 \%$ & $-120,2$ & $1.108,9$ & $3.438,4$ & $2.810,8$ & $5.755,2$ & $4.379,6$ & $6.102,6$ & $13.365,8$ & $3.940,0$ & $4.018,2$ & $6.143,5$ & $14.288,8$ & $65.231,7$ \\
\hline & $20-30 \%$ & $-423,4$ & $1.843,0$ & $3.162,1$ & $4.918,9$ & $4.643,4$ & $4.229,1$ & $8.951,0$ & $10.877,9$ & $16.350,7$ & $13.078,2$ & 935,0 & 22,5 & $68.588,2$ \\
\hline & $30-40 \%$ & $-202,8$ & $2.812,6$ & $6.622,1$ & $4.523,9$ & $6.286,8$ & $7.257,6$ & $14.112,2$ & $13.476,3$ & $19.442,1$ & $11.602,9$ & $13.575,8$ & $1.162,1$ & $100.671,7$ \\
\hline & $40-50 \%$ & $-320,4$ & $2.101,1$ & $5.244,2$ & $5.004,2$ & $4.617,0$ & $5.616,9$ & $14.910,8$ & $17.296,3$ & $20.852,2$ & $16.902,9$ & $1.332,8$ & $24.697,3$ & $118.255,3$ \\
\hline & $50-60 \%$ & $-586,5$ & $-846,9$ & $2.813,7$ & $3.881,3$ & $4.006,3$ & $11.664,9$ & $11.952,8$ & $30.173,8$ & $23.782,7$ & $35.659,5$ & $36.621,5$ & $84.316,5$ & $243.439,6$ \\
\hline & $60-70 \%$ & $-277,6$ & $2.995,3$ & $1.796,6$ & $4.106,7$ & $5.243,5$ & $12.209,5$ & $10.849,4$ & $18.911,5$ & $51.471,0$ & $23.820,3$ & $81.313,3$ & $42.908,4$ & $255.348,0$ \\
\hline & $70-80 \%$ & 24,8 & $1.632,0$ & $3.268,0$ & 224,4 & $8.130,9$ & $10.082,4$ & $10.238,1$ & $20.954,2$ & $51.547,6$ & $24.405,8$ & $39.764,6$ & $69.440,1$ & $239.713,0$ \\
\hline & $80-90 \%$ & $-9,2$ & $1.442,6$ & $5.321,4$ & $5.995,2$ & $9.411,3$ & $12.203,5$ & $13.454,3$ & $26.975,6$ & $52.723,6$ & $65.774,0$ & $63.632,6$ & $62.213,6$ & $319.138,5$ \\
\hline & 90-95\% & 2,8 & 145,7 & 372,0 & $2.995,2$ & $3.096,9$ & $3.496,5$ & $7.193,1$ & $15.628,6$ & $50.717,5$ & $48.375,8$ & $73.004,2$ & $80.343,0$ & $285.371,4$ \\
\hline & $95-98 \%$ & 0,2 & 183,8 & $2.760,1$ & $1.255,9$ & 157,9 & $4.213,2$ & $3.492,6$ & $4.426,4$ & $36.173,9$ & $37.313,6$ & $46.585,6$ & $137.067,7$ & $273.631,0$ \\
\hline & $98-100 \%$ & 0,0 & 212,7 & 133,9 & 79,0 & $1.659,5$ & $3.064,5$ & $1.134,4$ & $2.270,0$ & $8.218,3$ & $46.885,4$ & $62.583,3$ & $429.428,0$ & $555.668,9$ \\
\hline & Total & $-2.179,7$ & $11.395,3$ & $8.497,8$ & $9.051,3$ & $4.445,2$ & $30.192,2$ & $07.134,7$ & 17.352,4 & $39.583,6$ & $32.828,6$ & $429.981,5$ & $952.150,0$ & $2.560 .433,1$ \\
\hline
\end{tabular}

Fuente: Encuesta Financiera de las Familias 2014 y elaboración propia

\section{Cuadro 6. Distribución de la renta y la riqueza en el Reino de España. Valores de renta en millones de euros.}

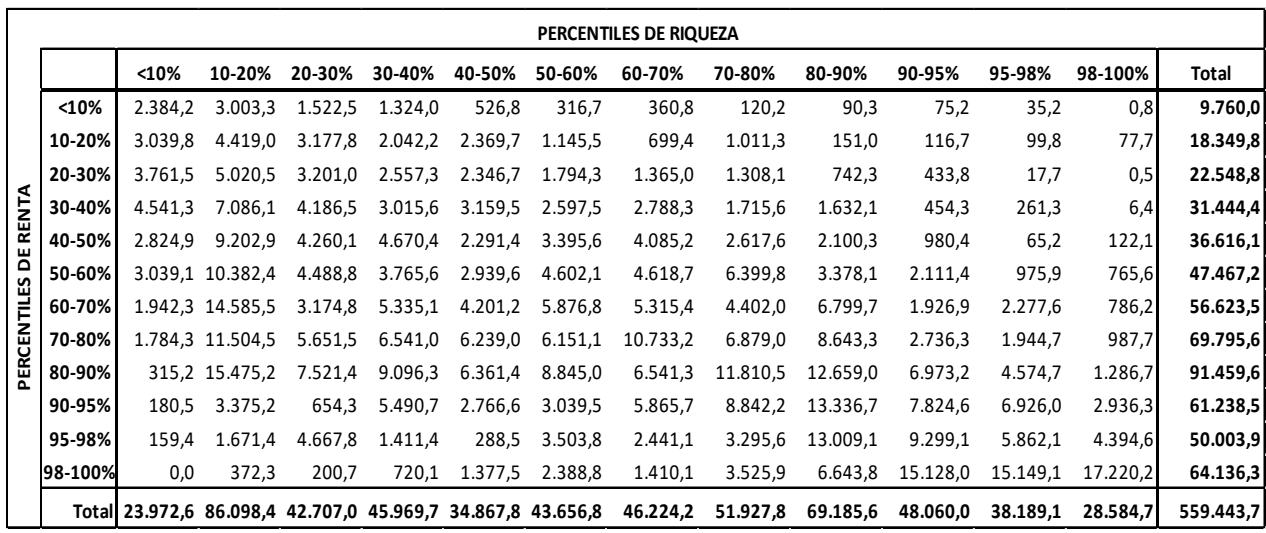

Fuente: Encuesta Financiera de las Familias 2014 y elaboración propia

de renta. Un tipo único del $2 \%{ }^{21}$ aplicado únicamente a los poseedores de la

21. Aunque no es exactamente lo mismo, pero si se toma en consideración el actual impuesto sobre el patrimonio existente en el Reino de España, con un tipo marginal máximo del $2,5 \%$, y en el que al menos 5 comunidades autónomas lo han situado ya en el $3 \%$, se tiene una clara justificación a esta propuesta de un tipo único del $2 \%$. No obstante, si se compara con puntos de vista más radicales como los que se aconsejan en el trabajo riqueza situados en el percentil 90-100, que repetimos acumulan cerca del billón de euros, permitiría recaudar una cifra superior a los 19.000 millones de euros, claramente superior al coste adicional de compensación (15.107 millones de euros) al que antes nos hemos referido en el apartado de resultados.

citado de Piketty, ciertamente esta cifra del 2\% debería considerarse muy moderada. 


\section{Conclusiones}

Esperamos haber mostrado cómo una RB puede financiarse a partir de los recursos disponibles. No hemos mencionado otras fuentes que podrían ayudar a la financiación como impuestos a las grandes fortunas, restricciones mucho más decididas que las actuales a la evasión y fraude fiscales, entre otras. Pero vamos a ser más concretos. Una imposición fuerte a las grandes fortunas es algo que nos parece elemental en una sociedad democrática. Incompresiblemente, hay quien considera una propuesta estrambótica este tipo de impuestos. Hay precedentes. En 1942, el presidente de los EEUU F.D. Roosevelt defendió una tasa marginal impositiva del $100 \%$ a quienes tuvieran unas rentas superiores a los 25.000 dólares anuales (es decir, unos 400.000 dólares actuales). No logró su objetivo, pero poco después en
EEUU se pudo imponer una tasa del $94 \%$ a las fortunas por encima de los 200.000 dólares. Solamente es un ejemplo.

Finalmente, para los que repiten sin cesar que financiar una RB es muy caro, solamente nos permitimos, además de los números mostrados, volverles la objeción: ¿cuánto cuesta no disponer de una RB? ¿Cómo valorar en costes las capacidades perdidas, el sufrimiento que se traduce en un incremento del deterioro de la salud mental, o en la imposibilidad de llevar vidas mínimamente valoradas de forma que valga la pena vivirlas...? Para ser cumplidos con la razón, estos costes, por decirlo de una manera fría, son inmensos. Nosotros hemos querido aquí mostrar algo mucho más modesto: cómo se puede financiar una RB de forma que toda la población tenga una existencia material garantizada.

\section{Glosario}

- $T U=\frac{\sum(R B+I R P F a-A H O R R O)}{\sum(\text { Renta Bruta }- \text { AHORRO })}$

- Renta neta ex - ante = Renta Bruta - CSS - IRPFa - IPPF - TF2

- IRPFP = TU (Renta Bruta positiva - AHORRO)

$T U=$ Tipo único

$R B=$ Renta Básica

IRPFa $=$ IRFF ex - ante

$A H O R R O=$ Ahorro al implementar la RB

CSS = Cotizaciones SS

$I P P F=$ Impuesto del patrimonio

TF2 $=$ Transferencias a otros hogares

IRPFP $=$ IRPF ex - post

- Renta neta ex - post antes compensación $=$ Renta Bruta - AHORRO + RB - IRPFP - CSS - IPPF - TF2

Agregación por hogares

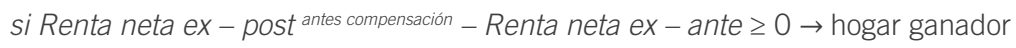

si Renta neta ex - post antes compensación - Renta neta ex - ante $<0 \rightarrow$ hogar perdedor

- Compensación = Todos los hogares por debajo del 80 percentil de Renta Bruta equivalente perdedores

- Renta neta ex - post $=\left\{\begin{array}{l}\text { Renta neta ex }- \text { post antes compensación si es ganador o perdedor sin compensación } \\ \text { Renta neta ex }- \text { ante }\end{array}\right.$ 


\section{Bibliografía}

Arcarons, J., D. Raventós, L. Torrens (2013a): Feasibility of Financing a Basic Income. Basic Income Studies, 9.

Arcarons, J., D. Raventós, L. Torrens. (2013b) Un modelo de financiación de la Renta Básica técnicamente factible y políticamente no inerte. Sin Permiso, 1 de diciembre de 2013. https://www. sinpermiso.info/textos/un-modelo-de-financiacin-de-la-renta-bsica-tcnicamentefactible-y-polticamente-no-inerte (última consulta 21/09/2020)

Arcarons, J., A. Domènech, D. Raventós, L. Torrens. (2014) Un modelo de financiación de la Renta Básica para el conjunto del Reino de España: sí, se puede y es racional. Sin Permiso, 7 de diciembre de 2014. https://www.sinpermiso.info/textos/ un-modelo-de-financiacin-de-la-rentabsica-para-el-conjunto-del-reino-deespaa-s-se-puede-y-es (última consulta 21/09/2020)

Arcarons, J., D. Raventós, L. Torrens (2016) La renda bàsica incondicional: una proposta racional per al segle xxi. Nota d'Economia, 103. pp 173-193. http://economia.gencat.cat/web/.content/70_economia_catalana/arxius/publicacions_periodiques/nota_d_economia/ ne_103/NE_103_c12.pdf (última consulta $21 / 09 / 2020$ )

Arcarons, J., D. Raventós, L. Torrens (2017a) ¡No son 188.000 millones! Los torpes errores del informe del BBVA sobre la Renta Básica. Sin Permiso, 23 de abril de 2017. https://www.sinpermiso.info/ textos/no-son-188000-millones-los-torpes-errores-del-informe-del-bbva-sobrela-renta-basica (última consulta 21/09/2020).

Arcarons, J., D. Raventós, L. Torrens (2017b) Renta básica incondicional.
Una propuesta de financiación racional y justa. Barcelona, Ediciones del Serbal.

Arcarons, J., D. Raventós, L. Torrens (2020). Nuevos modelos para financiar una renta básica incondicional y universal. Sin Permiso, 14 de junio de 2020. https://www.sinpermiso.info/textos/nuevos-modelos-para-financiar-una-rentabasica-incondicional-y-universal (última consulta 21/09/2020).

Arcarons, J., D. Raventós, L. Torrens (2020b). Aproximaciones con nuevos datos sobre cómo se podrían financiar asignaciones públicas: mención especial a la vivienda. Sin Permiso, 28 de junio de 2020. https://www.sinpermiso.info/textos/ aproximaciones-con-nuevos-datos-sobrecomo-se-podrian-financiar-asignaciones-publicas-mencion (última consulta 21/09/2020).

Bertomeu, M.J. y D. Raventós, D. (2020) Renta Básica y Renta Máxima: una concepción republicano-democrática. Daimon. Revista Internacional de Filosofía, 81. pp. 197-213.

Díaz, J.; Gimeno, J. A.; Gómez, V. (2019): Modelos de financiación de una renta básica para España. Revista Diecisiete. N ${ }^{\circ}$ 1, octubre; 135-159

Franco, H. et alters (2015) Nuevas propuestas para la tributación de la Riqueza en Europa. Diputación Foral de Gipuzkoa. Departamento de Hacienda y Finanzas. https://www.gipuzkoa.eus/ documents/2456431/2723083/Nuevas \%2Bpropuestas\%2Btributaci\%C3\%B3n \%2Bde\%2Bla\%2Briqueza\%2Ben\%2B Europa.pdf/fbdOb17a-5295-6726-af06aaa65c308640 (última consulta 21/09/2020).

Gentilini, U. et alters (2020) Exploring Universal Basic Income. International for Reconstruction and Development / The World Bank. http://documents1.worldbank.org/ curated/en/993911574784667955/pdf/ 
Exploring-Universal-Basic-Income-AGuide-to-Navigating-Concepts-Evidence-and-Practices.pdf (última consulta 21/09/2020).

Granell, R., A. Fuenmayor (2019): Implementing a Negative Income Tax. Net Cost, Poverty and Inequality Effects. Hacienda Pública Española / Review of Public Economics, 228-(1/2019); 83-108.

Gimeno, J.A. (2015): Aproximación a una renta básica sostenible. En Ayala, L. y J. Ruiz Huerta (eds.): Segundo informe sobre la desigualdad en España. Fundación Alternativas, Madrid, 97-103.

Kucharz, T. (2020) La gran oportunidad para un impuesto a la riqueza. CuartoPoder, 8 de mayo de 2020. https://www. cuartopoder.es/ideas/2020/05/08/lagran-oportunidad-para-un-impuesto-a-lariqueza/ (última consulta 21/09/2020).

Ortiz, I. et alters (2018) Universal Basic Income proposals in light of ILO standards: Key issues and global costing. Extension of Social Security-Working Paper, 62. Social Protection Department. International Labour Office. Geneva. https:// www.ilo.org/wcmsp5/groups/public/---ed_ protect/---soc_sec/documents/publication/wcms_648602.pdf (última consulta 21/09/2020).

Pettit, P. (2012) On the People's Terms: $A$ Republican Theory and Model of Democracy, Cambridge, Cambridge University Press.

Piketty, T. (2019). Capital e Ideología. Bilbao, Ediciones Deusto. 